2011

\title{
Young Radio Pulsars In Galactic Globular Clusters
}

J. Boyles

D. R. Lorimer

P.J. Turk

R. Mnatsakanov

R. S. Lynch

See next page for additional authors

Follow this and additional works at: https://researchrepository.wvu.edu/faculty_publications

\section{Digital Commons Citation}

Boyles, J.; Lorimer, D. R.; Turk, P. J.; Mnatsakanov, R.; Lynch, R. S.; Ransom, S. M.; Freire, P. C.; and Belczynski, K., "Young Radio Pulsars In Galactic Globular Clusters" (2011). Faculty Scholarship. 737.

https://researchrepository.wvu.edu/faculty_publications/737 
Authors

J. Boyles, D. R. Lorimer, P. J. Turk, R. Mnatsakanov, R. S. Lynch, S. M. Ransom, P. C. Freire, and K. Belczynski 


\title{
YOUNG RADIO PULSARS IN GALACTIC GLOBULAR CLUSTERS
}

\author{
J. Boyles ${ }^{1}$, D. R. Lorimer ${ }^{1}$, P. J. Turk ${ }^{2}$, R. Mnatsakanov ${ }^{2}$, R. S. LynCH ${ }^{3}$, S. M. Ransom ${ }^{4}$, P. C. Freire ${ }^{5}$, And K. Belczynski ${ }^{6}$ \\ ${ }^{1}$ Department of Physics, 210 Hodges Hall, West Virginia University, Morgantown, WV 26506, USA \\ ${ }^{2}$ Department of Statistics, 423 Hodges Hall, West Virginia University, Morgantown, WV 26506, USA \\ ${ }^{3}$ Department of Astronomy, University of Virginia, P.O. Box 400325, Charlottesville, VA 22904, USA \\ ${ }^{4}$ National Radio Astronomy Observatory, 520 Edgemont Rd, Charlottesville, VA, USA \\ ${ }^{5}$ Max-Planck-Institut für Radioastronomie, Auf dem Hügel D-53121, Bonn, Germany \\ ${ }^{6}$ Astronomical Observatory, University of Warsaw, AL Ujazdowskie 4,00-478, Warsaw, Poland \\ Received 2011 May 3; accepted 2011 August 18; published 2011 November 3
}

\begin{abstract}
Currently three isolated radio pulsars and one binary radio pulsar with no evidence of any previous recycling are known in 97 surveyed Galactic globular clusters (GCs). As pointed out by Lyne et al., the presence of these pulsars cannot be explained by core-collapse supernovae, as commonly assumed for their counterparts in the Galactic disk. We apply a Bayesian analysis to the results from surveys for radio pulsars in GCs and find the number of potentially observable non-recycled radio pulsars present in all clusters to be $<3600$. Accounting for beaming and retention considerations, the implied birthrate for any formation scenario for all 97 clusters is $<0.25$ pulsars century ${ }^{-1}$ assuming a Maxwellian distribution of velocities with a dispersion of $10 \mathrm{~km} \mathrm{~s}^{-1}$. The implied birthrates for higher velocity dispersions are substantially higher than inferred for such pulsars in the Galactic disk. This suggests that the velocity dispersion of young pulsars in GCs is significantly lower than those of disk pulsars. These numbers may be substantial overestimates due to the fact that the currently known sample of young pulsars is observed only in metal-rich clusters. We propose that young pulsars may only be formed in GCs with metallicities with $\log [\mathrm{Fe} / \mathrm{H}]>-0.6$. In this case, the potentially observable population of such young pulsars is $447_{-399}^{+1420}$ (the error bars give a 95\% confidence interval) and their birthrate is $0.012_{-0.010}^{+0.037}$ pulsars century ${ }^{-1}$. The most likely creation scenario to explain these pulsars is the electron capture supernova of an $\mathrm{OMgNe}$ white dwarf.
\end{abstract}

Key words: globular clusters: general - methods: statistical - pulsars: general

Online-only material: color figures

\section{INTRODUCTION}

Since the first discovery of PSR B1821-24 in M28 (Lyne et al. 1987), 143 pulsars $^{7}$ have been discovered in 27 globular clusters (GCs). While the majority of GC pulsars are thought to have been formed in low-mass X-ray binary systems, in which the neutron star has been spun up to millisecond periods by the transfer of matter from an evolved companion (see, e.g., Camilo \& Rasio 2005 for a recent review), a small minority of the GC pulsar population appear to be similar to the isolated "normal" pulsars which inhabit the disk of our Galaxy (i.e., spin periods $(P)$ of several hundred millisecond, characteristic ages $\left(\tau_{c}\right)$ of $10^{7}-10^{8} \mathrm{yr}$, and inferred dipole magnetic field strengths $(B)$ of $\left.10^{11}-10^{12} \mathrm{G}\right)$. These pulsars were originally discussed by Lyne et al. (1996) who noted that, since no significant star formation has occurred in GCs in the last billion years (Briley et al. 1994), this population appears to be highly anomalous. Lyne et al. (1996) also note that the young pulsars appear in metalrich GCs. This trend has persisted despite 15 years of intense searches of most of the cluster population. A goal of this work is to investigate the statistical and astrophysical significance of this result.

Recent improvements in observational systems over the past decade have led to a wealth of discoveries of pulsars in GCs. Sensitive radio surveys for pulsars have been conducted on almost 100 GCs to date. The two most fruitful have been Terzan 5 (Ransom et al. 2005) and 47 Tucanae (Freire et al. 2001) with 34 and 23 pulsars, respectively. Neither of these

\footnotetext{
7 For an up-to-date list of known globular cluster pulsars, see http://www.naic.edu/ pfreire/GCpsr.html
}

clusters harbor any normal pulsars, which we will henceforth define as being pulsars with spin periods $P>100 \mathrm{~ms}$ and inferred magnetic field strengths $B>10^{11} \mathrm{G}$. This is somewhat surprising, given that the significant selection effects known to hamper the detection of binary and millisecond pulsars (MSPs) in clusters (Camilo \& Rasio 2005) are not as severe for normal isolated pulsars.

Clearly, some physical mechanism is at work which produces these apparently young pulsars in GCs in a different way to how we believe they are formed in the disk of our Galaxy. One possible method is the collapse of a white dwarf via an electron capture supernova (ECS) in a binary or collisional system (Ivanova et al. 2008). In this paper, we revisit the statistics of the normal pulsars based on the results of recent surveys and recent studies of the Galactic population of normal pulsars (Faucher-Giguère \& Kaspi 2006, hereafter FK06; Ridley \& Lorimer 2010). In Section 2, we review the current sample of normal pulsars in GCs. In Section 3, we compile a list of flux density detection limits for 97 GCs based on published searches and some recent unpublished results. In Section 4, we use these limits to characterize the population of young pulsars. In Section 5, we discuss the intrinsic population and birthrate of young pulsars in GCs. In Section 6, we discuss formation scenarios and future work with young GC pulsars.

\section{THE CURRENT SAMPLE OF YOUNG PULSARS IN GCs}

At least three, possibly four, young pulsars are known in two or three GCs. These four pulsars are B1718-19 in NGC 6342, B 1745-20 in NGC 6640, and J1823-3021B and J1823-3021C in NGC 6624. The properties of these pulsars are summarized in 
Table 1

The Four Young Pulsars Currently Known in GCs

\begin{tabular}{|c|c|c|c|c|c|c|c|c|c|}
\hline PSR & Cluster & $\begin{array}{c}P \\
(\mathrm{~ms})\end{array}$ & $\begin{array}{c}\dot{P} \\
\left(\mathrm{~s} \mathrm{~s}^{-1}\right)\end{array}$ & $\begin{array}{c}\mathrm{DM} \\
\left(\mathrm{pc} \mathrm{cm}^{-3}\right)\end{array}$ & $\begin{array}{c}B \\
(\mathrm{G})\end{array}$ & $\begin{array}{c}\tau_{c} \\
(\mathrm{yr})\end{array}$ & $\begin{array}{l}S_{1400} \\
(\mathrm{mJy})\end{array}$ & Binary? & $\begin{array}{l}\text { Timing Solution } \\
\text { Reference }\end{array}$ \\
\hline B $1718-19$ & NGC 6342 & 1004 & $1.6 \times 10^{-15}$ & 75.7 & $1.3 \times 10^{12}$ & $9.8 \times 10^{6}$ & 0.30 & Yes & Lyne et al. (1993) \\
\hline B $1745-20$ & NGC 6440 & 288 & $4.0 \times 10^{-16}$ & 219.4 & $3.4 \times 10^{11}$ & $1.1 \times 10^{7}$ & 0.37 & No & Freire et al. (2008) \\
\hline $\mathrm{J} 1823-3021 \mathrm{~B}$ & NGC 6624 & 379 & $3.0 \times 10^{-17}$ & 86.9 & $1.1 \times 10^{11}$ & $2.0 \times 10^{8}$ & 1.04 & No & Lynch et al. (2011) \\
\hline $\mathrm{J} 1823-3021 \mathrm{C}$ & NGC 6624 & 406 & $2.2 \times 10^{-16}$ & 86.7 & $3.0 \times 10^{11}$ & $2.9 \times 10^{7}$ & 0.71 & No & Lynch et al. (2011) \\
\hline
\end{tabular}

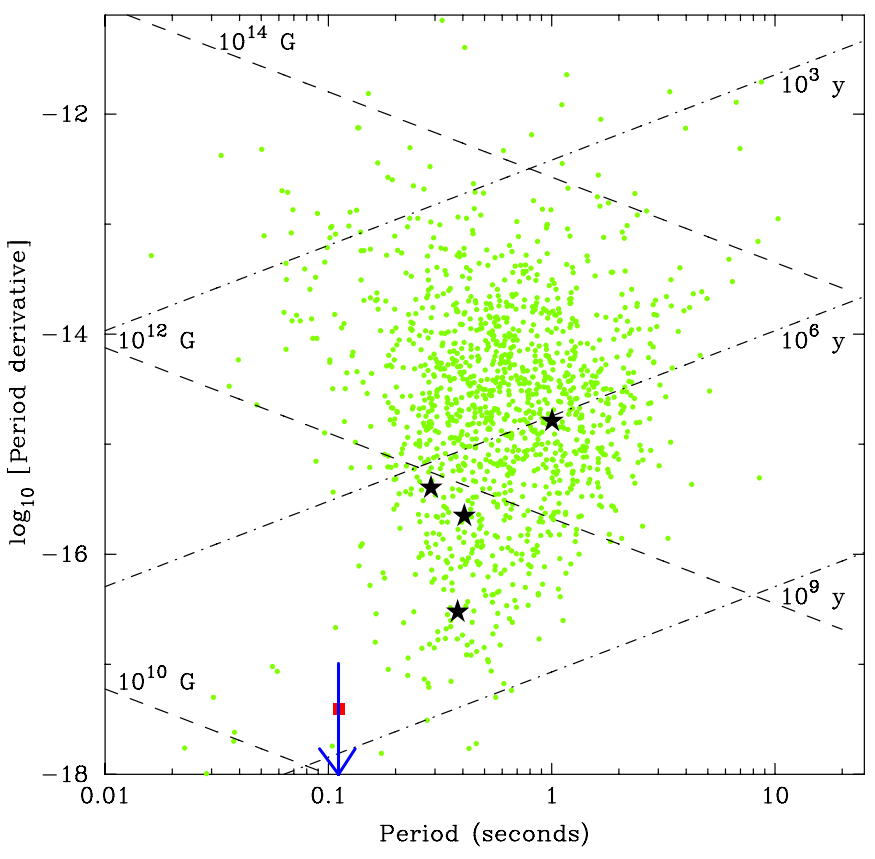

Figure 1. $P-\dot{P}$ diagram showing young GC pulsars as black stars and PSR $\mathrm{J} 1750-37 \mathrm{~A}$ as a red square. PSR B2127+11A is shown as a blue arrow with the arrow representing the limit on period derivative at the bottom of the diagram. Dashed lines are lines of constant magnetic field and dot-dashed lines are lines of constant characteristic age. The pulsar population data are taken from the Australia Telescope National Facility pulsar database (Manchester et al. 2005). (A color version of this figure is available in the online journal.)

Table 1 and their GC properties relevant to this data analysis can be seen in Table 3. As can be seen by inspecting their position with respect to other normal Galactic field pulsars on the $P-\dot{P}$ diagram shown in Figure 1, these pulsars appear to be consistent with the distribution of normal pulsars in the Galactic disk.

Two long-period pulsars are not included in this compilation. First, the 110 ms pulsar B2127+11A in GC NGC 7078 which is known to have a negative period derivative due to contamination by the cluster potential and nearby stars (Wolszczan et al. 1989). As discussed by these authors, the pulsar most likely has a small intrinsic period derivative indicating that it has undergone some recycling in a binary system which has subsequently been disrupted due to close encounters in the cluster. It is also noted that a recent measurement of the second period derivative of $\ddot{P}=3.2 \times 10^{-29} \mathrm{~s}^{-1}$ for this pulsar is entirely consistent with encounters with nearby stars in the cluster (Jacoby et al. 2006). Another long-period pulsar which we do not consider to be young is the 110 ms pulsar J1750-37A in GC NGC 6441 (Freire et al. 2008). This is a member of an eccentric binary system and its low-period derivative places it closer to the region of the $P-\dot{P}$ diagram occupied by the double neutron star binary systems and the eccentric neutron-star-white-dwarf binaries (see Figure 1).
One other caveat needs to be discussed with the sample of young pulsars present in this paper. PSR B1718-19 may not truly be a member of NGC 6342 and is the only pulsar located near NGC 6342. An earlier discussion of this subject is presented in Bailes et al. (2005) and a further expansion will be presented here. The original paper used PSR B1718-19's position, binary status, and dispersion measure (DM) to argue for its association with NGC 6342. Its position of $2 ! 3$ away from the cluster's center is three times greater than the halfmass radius of the cluster. All other young pulsars presented in Table 1 and a majority of all GC pulsars with timing solutions are found within the half-mass radius. The DM of $75.7 \mathrm{pc} \mathrm{cm}^{-3}$ for PSR B1718-19 is somewhat below the predicted values of $120 \mathrm{pc} \mathrm{cm}^{-3}$ using $\mathrm{DM}=20 / \sin (b) \mathrm{pc} \mathrm{cm}^{-3}$ (Lyne et al. 1995), where $b=9.72,130 \mathrm{pc} \mathrm{cm}^{-3}$ (Taylor \& Cordes 1993), or $229 \mathrm{pc} \mathrm{cm}^{-3}$ (Cordes \& Lazio 2002). These DM models are uncertain, but factors in the range of 1.5 to 3 are larger than the known differences for sources within $|b|<10^{\circ}$ of the Galactic plane. The unusual binary nature of PSR B1718-19 gives support to its association with NGC 6342 due to its existence as an eclipsing low-mass binary pulsar (Freire 2005) and due to the fact that the proportion of pulsars with binary companions is about two orders of magnitude greater for GC pulsars than Galactic field pulsars (Lyne et al. 1995). In the following sections, given the lack of clear evidence for or against the association, the results are discussed both with and without the inclusion of PSR B1718-19 and will be explicitly stated when PSR B1718-19 is not included.

\section{SENSITIVITY LIMITS FOR YOUNG PULSARS IN GCs}

To carry out the statistical analysis of this sample, it is necessary to have a substantial compilation of upper limits from GCs that have been searched for such pulsars. Table 3 gives our compilation of flux density limits at $1400 \mathrm{MHz}$ for searches of each cluster, $S_{\min }$ and GC properties relevant to this work. The main surveys that were used are Hessels et al. (2007), Lynch \& Ransom (2011), Lynch et al. (2011), and A. Possenti (2010, unpublished). The rest of the GCs had their flux limits taken from the most recent discovery paper. If the paper did not quote a flux limit, one was derived using the survey parameters from the paper using the radiometer equation for pulsars which gives

$$
S_{\min }=\frac{\beta\left(\mathrm{S} / \mathrm{N}_{\min }\right) T_{\text {sys }}}{G \sqrt{n_{\mathrm{p}} t_{\mathrm{int}} \Delta f}} \sqrt{\frac{\delta}{1-\delta}},
$$

where $\beta$ is a digitization correction faction, $\mathrm{S} / \mathrm{N}_{\min }$ is the minimum signal-to-noise ratio, $T_{\text {sys }}$ is the system temperature of the telescope, $G$ is the gain of the telescope, $n_{\mathrm{p}}$ is the number of polarizations summed, $t_{\text {int }}$ is the observation time, $\Delta f$ is the bandwidth of the backend, and $\delta$ is the fractional pulse width (Lorimer \& Kramer 2004).

The parameters $t_{\mathrm{int}}, \Delta f$, and $n_{\mathrm{p}}$ are all taken from the relevant survey paper. The values for $G$ and $\beta$ can be obtained from 
Table 2

Parameters for Bulge Globular Clusters

\begin{tabular}{|c|c|c|c|c|c|c|c|c|}
\hline $\begin{array}{l}\text { Globular } \\
\text { Cluster }\end{array}$ & $\begin{array}{c}l \\
\left({ }^{o}\right)\end{array}$ & $\begin{array}{c}b \\
\left({ }^{o}\right)\end{array}$ & $\begin{array}{l}\text { Distance } \\
(\mathrm{kpc})\end{array}$ & $\begin{array}{l}\text { Distance from Galactic Center } \\
\qquad(\mathrm{kpc})\end{array}$ & $\begin{array}{c}r_{t} \\
(\mathrm{pc})\end{array}$ & $\begin{array}{c}S_{\min } \\
\left(\mathrm{mJy} \mathrm{pc}^{2}\right)\end{array}$ & $\begin{array}{l}\text { Mass } \\
\left(M_{\odot}\right)\end{array}$ & $\begin{array}{c}V_{\sigma} \\
\left(\mathrm{km} \mathrm{s}^{-1}\right)\end{array}$ \\
\hline NGC 6325 & 0.97 & 8.00 & 7.8 & 1.1 & 15.8 & 54.8 & 223,000 & 5.9 \\
\hline NGC 6355 & 359.59 & 5.43 & 9.2 & 1.4 & 31.6 & 77.5 & 252,000 & $\mathrm{~N} / \mathrm{A}$ \\
\hline Terzan 2 & 356.32 & 2.30 & 7.5 & 0.8 & 24.0 & none & 3,290 & $\mathrm{~N} / \mathrm{A}$ \\
\hline Terzan 4 & 356.02 & 1.31 & 7.2 & 1.0 & 23.3 & none & $\mathrm{N} / \mathrm{A}$ & $\mathrm{N} / \mathrm{A}$ \\
\hline HP 1 & 357.44 & 2.12 & 8.2 & 0.5 & 20.4 & none & 95,700 & $\mathrm{~N} / \mathrm{A}$ \\
\hline Liller 1 & 354.84 & -0.16 & 8.2 & 0.8 & 36.5 & 9.9 & 289,000 & $\mathrm{~N} / \mathrm{A}$ \\
\hline Terzan 1 & 357.57 & 1.00 & 6.7 & 1.3 & 22.8 & 77.5 & 5,360 & $\mathrm{~N} / \mathrm{A}$ \\
\hline Ton 2 & 350.80 & -3.42 & 8.2 & 1.4 & 25.3 & none & 7,330 & $\mathrm{~N} / \mathrm{A}$ \\
\hline Terzan 5 & 3.84 & 1.69 & 6.9 & 1.2 & 21.9 & 11.6 & 374,000 & $\mathrm{~N} / \mathrm{A}$ \\
\hline NGC 6440 & 7.73 & 3.80 & 8.5 & 1.3 & 13.0 & 9.8 & 811,000 & $\mathrm{~N} / \mathrm{A}$ \\
\hline Terzan 6 & 358.57 & -2.16 & 6.8 & 1.3 & 43.6 & 7.3 & 300,000 & $\mathrm{~N} / \mathrm{A}$ \\
\hline UKS 1 & 5.13 & 0.76 & 7.8 & 0.7 & 45.6 & 54.8 & 145,000 & $\mathrm{~N} / \mathrm{A}$ \\
\hline Terzan 9 & 3.61 & -1.99 & 7.1 & 1.1 & 21.3 & 17.0 & 9,570 & $\mathrm{~N} / \mathrm{A}$ \\
\hline NGC 6522 & 1.02 & -3.93 & 7.7 & 0.6 & 34.8 & 54.8 & 300,000 & 6.7 \\
\hline NGC 6528 & 1.14 & -4.17 & 7.9 & 0.6 & 33.1 & 54.8 & 152,000 & $\mathrm{~N} / \mathrm{A}$ \\
\hline NGC 6558 & 0.20 & -6.02 & 7.4 & 1.0 & 28.5 & 63.3 & 98,400 & 3.1 \\
\hline NGC 6624 & 2.79 & -7.91 & 7.9 & 1.2 & 47.4 & 16.0 & 257,000 & 5.4 \\
\hline
\end{tabular}

the websites or papers that give the telescope and back-end specifications. $\mathbf{S} / \mathrm{N}_{\min }$ and $\delta$ are parameters that are chosen to have values of 8 and 0.1 , respectively. $T_{\text {sys }}$ can be expressed as

$$
T_{\mathrm{sys}}=T_{\mathrm{rec}}+T_{\mathrm{CMB}}+T_{\mathrm{gsyn}}+T_{\mathrm{spill}}+T_{\mathrm{RFI}},
$$

where $T_{\text {rec }}$ is the receiver temperature, $T_{\mathrm{CMB}}$ is the cosmic background temperature, $T_{\text {gsyn }}$ is the Galactic synchrotron temperature, $T_{\text {spill }}$ is the spillover temperature from sources in the side lobes of the telescope, and $T_{\mathrm{RFI}}$ is the increase in system temperature due to terrestrial radio frequency interference (RFI). The values of $T_{\mathrm{rec}}, T_{\mathrm{CMB}}$, and $T_{\mathrm{gsyn}}$ are well known, but $T_{\text {spill }}$ and $T_{\text {RFI }}$ can vary greatly with time and telescope position causing large uncertainties in $T_{\text {sys }}$ and can only truly be obtained by proper calibration. Due to pulsar survey observations rarely ever having accurate calibration, the expression for $T_{\text {sys }}$ has been simplified to include only the $T_{\mathrm{rec}}, T_{\mathrm{CMB}}$, and $T_{\mathrm{gsyn}}$. One last fact to mention is due to these pulsars having long periods, DM smearing and scattering are unlikely to be important and are not considered in this work.

For any surveys which were not carried out at $1400 \mathrm{MHz}$, the quoted limits are scaled from the observing frequency to $1400 \mathrm{MHz}$ using a simple power law $S \propto v^{-1.6}$, which is consistent with the average spectral behavior for a large sample of normal pulsars (Lorimer et al. 1995).

\section{THE POTENTIALLY OBSERVABLE POPULATION OF YOUNG PULSARS IN GCs}

For all clusters listed in Table 3, we can use the flux density limits to model the most likely number of potentially observable pulsars $^{8}$ in each cluster. Using a binomial data model, we carry out a simple Bayesian analysis described below. This method rests on a key simplifying assumption about the luminosity function of young pulsars. Following Faucher-Giguère \& Kaspi (2006), we will assume that the parent population follows a log-normal luminosity function defined (at $1400 \mathrm{MHz}$ ) to have a mean in the base-10 logarithm of $L$ (in $\mathrm{mJy}$ ) to be -1.1 and a standard deviation of 0.9 . This assumption is reasonable if

\footnotetext{
8 The estimates in this section do not account for beaming effects, or the fact that many pulsars will escape the cluster potential and will be missed by the surveys. We will discuss these issues in Section 5.
}

we consider that the spin-down evolution of isolated pulsars in GCs is the same as the Galactic disk. Variations on the mean and standard deviation of the log-normal luminosity function have been applied to our analysis using models from Ridley \& Lorimer (2010). These values are in the range of -1.04 to -1.19 for the mean of the luminosity function in the base- 10 and in the range of 0.91 to 0.98 for the standard deviation.

We can model the number of pulsars in a particular cluster, $N$, given a "detection probability" $\theta$ and an observed sample of $n$ pulsars. Bayes' theorem gives the joint posterior probability density for $N$ and $\theta$ as

$$
p(N, \theta \mid n) \propto p(n \mid N, \theta) p(N, \theta) .
$$

Here $p(n \mid N, \theta)$ is the probability of observing $n$ pulsars from a parent population of $N$ with some $\theta$ and $p(N, \theta)$ is the joint prior probability density for $N$ and $\theta$. The prior distribution for $N$ is assumed to be uniform in the range $n$ to $\infty$. Graphically, $\theta$ is the ratio of the area under the luminosity function for $L>L_{\min }$ to the total area under the function. We evaluate $\theta$ numerically using Monte Carlo integration of the FaucherGiguère \& Kaspi (2006) luminosity function and four luminosity functions from Ridley \& Lorimer (2010). The prior distribution for $\theta$ is assumed to be independent of $N$ and uniform in the range $\theta_{\min }$ to $\theta_{\max }$, which are defined to be the minimum and maximum probabilities from the five distribution functions. The simplest choice for a likelihood function is the binomial distribution, i.e.,

$$
p(n \mid N, \theta)=\frac{N !}{n !(N-n) !} \theta^{n}(1-\theta)^{N-n},
$$

where $\theta$ depends on the luminosity limit and the assumed parent luminosity function.

For the majority of cases in which there are no detections in a cluster, $n=0$ and the likelihood term simplifies considerably to

$$
p(n \mid N, \theta)=(1-\theta)^{N} \text {. }
$$

For the cases of NGC 6440 and NGC 6342, where there is one detection, i.e., $n=1$, we have

$$
p(n \mid N, \theta)=N \theta(1-\theta)^{N-1},
$$


Table 3

Parameters for Globular Clusters Searched for Pulsars (Globular Cluster Parameters from Harris (1996))

\begin{tabular}{|c|c|c|c|c|c|c|c|}
\hline $\begin{array}{l}\text { Globular } \\
\text { Cluster }\end{array}$ & $\begin{array}{l}\text { Distance } \\
(\mathrm{kpc})\end{array}$ & $\begin{array}{c}S_{\min }(1400) \\
(\mu \mathrm{Jy})\end{array}$ & $\begin{array}{c}V_{\mathrm{esc}} \\
\left(\mathrm{km} \mathrm{s}^{-1}\right)\end{array}$ & $\begin{array}{l}\text { Metallicity } \\
\log [\mathrm{Fe} / \mathrm{H}]\end{array}$ & $\begin{array}{l}\text { Mass } \\
\left(M_{\odot}\right)\end{array}$ & $\begin{array}{c}\text { Number of } \\
\text { Young Pulsars }\end{array}$ & $\begin{array}{c}\text { Search } \\
\text { Reference }\end{array}$ \\
\hline 47 Tuc & 4.5 & 167.6 & 68.8 & -0.72 & $1,500,000$ & 0 & Freire et al. 2001 \\
\hline NGC 288 & 8.9 & 6.5 & 13.3 & -1.32 & 112,000 & 0 & Lynch \& Ransom 2011 \\
\hline NGC 1261 & 16.3 & 38.8 & 3.4 & -1.27 & 341,000 & 0 & A. Possenti 2010, unpublished \\
\hline Pal 2 & 27.2 & 34 & 27.8 & -1.42 & 410,000 & 0 & Hessels et al. 2007 \\
\hline NGC 1851 & 12.1 & 30.1 & 51.8 & -1.18 & 551,000 & 0 & Freire et al. 2004 \\
\hline NGC 2298 & 10.8 & 4.1 & 18.4 & -1.92 & 84,900 & 0 & Lynch \& Ransom 2011 \\
\hline NGC 2808 & 9.6 & 54.8 & 72.8 & -1.14 & $1,420,000$ & 0 & A. Possenti 2010, unpublished \\
\hline E3 & 8.1 & 54.8 & 3.0 & -0.83 & 3,290 & 0 & A. Possenti 2010, unpublished \\
\hline NGC 3201 & 4.9 & 54.8 & 22.0 & -1.59 & 254,000 & 0 & A. Possenti 2010, unpublished \\
\hline NGC 4147 & 19.3 & 19 & 18.3 & -1.80 & 74,700 & 0 & Hessels et al. 2007 \\
\hline NGC 4372 & 5.8 & 54.8 & 21.5 & -2.17 & 329,000 & 0 & A. Possenti 2010, unpublished \\
\hline NGC 4590 & 10.3 & 54.8 & 18.2 & -2.23 & 223,000 & 0 & A. Possenti 2010, unpublished \\
\hline NGC 4833 & 6.6 & 77.5 & 31.8 & -1.85 & 410,000 & 0 & A. Possenti 2010, unpublished \\
\hline NGC 5024 & 17.9 & 19 & 33.4 & -2.10 & 826,000 & 0 & Hessels et al. 2007 \\
\hline NGC 5053 & 17.4 & 20 & 8.9 & -2.27 & 125,000 & 0 & Hessels et al. 2007 \\
\hline NGC 5139 & 5.2 & 48.3 & 60.4 & -1.53 & $3,350,000$ & 0 & A. Possenti 2010, unpublished \\
\hline NGC 5272 & 10.2 & 21 & 37.2 & -1.50 & 957,000 & 0 & Hessels et al. 2007 \\
\hline NGC 5286 & 11.7 & 54.8 & 52.6 & -1.69 & 713,000 & 0 & A. Possenti 2010, unpublished \\
\hline NGC 5466 & 16.0 & 22 & 9.5 & -1.98 & 179,000 & 0 & Hessels et al. 2007 \\
\hline Pal 5 & 23.2 & 32 & 3.2 & -1.41 & 30,000 & 0 & Hessels et al. 2007 \\
\hline NGC 5897 & 12.5 & 5.5 & 13.4 & -1.90 & 211,000 & 0 & Lynch \& Ransom 2011 \\
\hline NGC 5904 & 7.5 & 25 & 47.7 & -1.29 & 857,000 & 0 & Hessels et al. 2007 \\
\hline NGC 5927 & 7.7 & 54.8 & 33.9 & -0.49 & 338,000 & 0 & A. Possenti 2010, unpublished \\
\hline NGC 5946 & 10.6 & 54.8 & 25.3 & -1.29 & 281,000 & 0 & A. Possenti 2010, unpublished \\
\hline NGC 5986 & 10.4 & 3.7 & 37.0 & -1.59 & 599,000 & 0 & Lynch \& Ransom 2011 \\
\hline M 80 & 10.0 & 5.7 & 48.7 & -1.75 & 502,000 & 0 & Lynch et al. 2011 \\
\hline NGC 6121 & 2.2 & 131.4 & 34.2 & -1.16 & 195,000 & 0 & Lyne et al. 1988 \\
\hline ESO452 & 8.3 & 54.8 & 5.9 & -1.50 & 75,000 & 0 & A. Possenti 2010, unpublished \\
\hline NGC 6144 & 8.9 & 54.8 & 14.1 & -1.76 & 169,000 & 0 & A. Possenti 2010, unpublished \\
\hline NGC 6139 & 10.1 & 77.5 & 59 & -1.65 & 566,000 & 0 & A. Possenti 2010, unpublished \\
\hline NGC 6171 & 6.4 & 54.8 & 25 & -1.02 & 182,000 & 0 & A. Possenti 2010, unpublished \\
\hline NGC 6205 & 7.1 & 27 & 39.1 & -1.53 & 775,000 & 0 & Hessels et al. 2007 \\
\hline NGC 6218 & 4.8 & 54.8 & 28.5 & -1.37 & 217,000 & 0 & A. Possenti 2010, unpublished \\
\hline NGC 6235 & 11.5 & 54.8 & 16.8 & -1.28 & 73,300 & 0 & A. Possenti 2010, unpublished \\
\hline NGC 6254 & 4.4 & 66.6 & 29.5 & -1.56 & 252,000 & 0 & A. Possenti 2010, unpublished \\
\hline Pal 15 & 45.1 & 38 & 4.3 & -2.07 & 40,300 & 0 & Hessels et al. 2007 \\
\hline NGC 6266 & 6.8 & 22.1 & 97.8 & -1.18 & $1,220,000$ & 0 & Chandler 2003 \\
\hline NGC 6273 & 8.8 & 54.8 & 58.4 & -1.74 & $1,100,000$ & 0 & A. Possenti 2010, unpublished \\
\hline NGC 6284 & 15.3 & 54.8 & 28.6 & -1.26 & 361,000 & 0 & A. Possenti 2010, unpublished \\
\hline NGC 6287 & 9.4 & 77.5 & 30.4 & -2.10 & 188,000 & 0 & A. Possenti 2010, unpublished \\
\hline NGC 6293 & 9.5 & 54.8 & 41.7 & -1.99 & 329,000 & 0 & A. Possenti 2010, unpublished \\
\hline NGC 6304 & 5.9 & 54.8 & 38.4 & -0.45 & 217,000 & 0 & A. Possenti 2010, unpublished \\
\hline M 92 & 8.3 & 5.1 & 47.1 & -2.31 & 489,000 & 0 & Lynch \& Ransom 2011 \\
\hline NGC 6325 & 7.8 & 54.8 & 42.5 & -1.25 & 223,000 & 0 & A. Possenti 2010, unpublished \\
\hline NGC 6333 & 7.9 & 54.8 & 37.7 & -1.77 & 422,000 & 0 & A. Possenti 2010, unpublished \\
\hline NGC 6342 & 8.5 & 270 & 22.9 & -0.55 & 96,600 & 1 & Biggs \& Lyne 1996 \\
\hline NGC 6355 & 9.2 & 77.5 & 40.3 & -1.37 & 252,000 & 0 & A. Possenti 2010 , unpublished \\
\hline Liller 1 & 8.2 & 9.9 & 41.2 & -0.33 & 289,000 & 0 & Lynch et al. 2011 \\
\hline Ter 1 & 6.7 & 77.5 & 7.4 & -1.03 & 5,360 & 0 & A. Possenti 2010, unpublished \\
\hline NGC 6388 & 9.9 & 54.8 & 124 & -0.55 & $2,170,000$ & 0 & A. Possenti 2010, unpublished \\
\hline NGC 6402 & 9.3 & 54.8 & 39.1 & -1.28 & $1,040,000$ & 0 & A. Possenti 2010, unpublished \\
\hline NGC 6401 & 10.6 & 77.5 & 38.3 & -1.02 & 286,000 & 0 & A. Possenti 2010, unpublished \\
\hline NGC 6397 & 2.3 & 31.5 & 48.3 & -2.02 & 115,000 & 0 & A. Possenti 2010, unpublished \\
\hline Pal 6 & 5.8 & 6.7 & 28.0 & -0.91 & 228,000 & 0 & Lynch et al. 2011 \\
\hline NGC 6426 & 20.6 & 25 & 14.7 & -2.15 & 117,000 & 0 & Hessels et al. 2007 \\
\hline Ter 5 & 6.9 & 11.6 & 50.5 & -0.23 & 374,000 & 0 & Ransom et al. 2005 \\
\hline NGC 6440 & 8.5 & 9.8 & 85.2 & -0.36 & 811,000 & 1 & Freire et al. 2008 \\
\hline NGC 6441 & 11.6 & 12.4 & 102 & -0.46 & $1,570,000$ & 0 & Freire et al. 2008 \\
\hline Ter 6 & 6.8 & 7.3 & 38.3 & -0.56 & 300,000 & 0 & Lynch et al. 2011 \\
\hline NGC 6453 & 11.6 & 80.4 & 22.4 & -1.50 & 169,000 & 0 & A. Possenti 2010, unpublished \\
\hline UKS 1 & 7.8 & 54.8 & 25.4 & -0.64 & 145,000 & 0 & A. Possenti 2010, unpublished \\
\hline NGC 6496 & 11.3 & 54.8 & 19.7 & -0.46 & 200,000 & 0 & A. Possenti 2010, unpublished \\
\hline Ter 9 & 7.1 & 17.0 & 9.8 & -1.05 & 9,570 & 0 & Lynch \& Ransom 2011 \\
\hline NGC 6517 & 10.6 & 3.0 & 82.9 & -1.23 & 526,000 & 0 & Lynch et al. 2011 \\
\hline NGC 6522 & 7.7 & 54.8 & 42.3 & -1.34 & 300,000 & 0 & A. Possenti 2010, unpublished \\
\hline
\end{tabular}


Table 3

(Continued)

\begin{tabular}{|c|c|c|c|c|c|c|c|}
\hline $\begin{array}{l}\text { Globular } \\
\text { Cluster }\end{array}$ & $\begin{array}{c}\text { Distance } \\
(\mathrm{kpc})\end{array}$ & $\begin{array}{c}S_{\min }(1400) \\
(\mu \mathrm{Jy})\end{array}$ & $\begin{array}{c}V_{\mathrm{esc}} \\
\left(\mathrm{km} \mathrm{s}^{-1}\right)\end{array}$ & $\begin{array}{l}\text { Metallicity } \\
\log [\mathrm{Fe} / \mathrm{H}]\end{array}$ & $\begin{array}{l}\text { Mass } \\
\left(M_{\odot}\right)\end{array}$ & $\begin{array}{c}\text { Number of } \\
\text { Young Pulsars }\end{array}$ & $\begin{array}{c}\text { Search } \\
\text { Reference }\end{array}$ \\
\hline NGC 6528 & 7.9 & 54.8 & 26.4 & -0.11 & 152,000 & 0 & A. Possenti 2010, unpublished \\
\hline NGC 6535 & 6.8 & 41 & 10.0 & -1.79 & 20,000 & 0 & Hessels et al. 2007 \\
\hline NGC 6539 & 7.8 & 47.0 & 35.8 & -0.63 & 536,000 & 0 & D’Amico et al. 1993 \\
\hline NGC 6540 & 5.3 & 77.5 & 27.6 & -1.35 & 36,400 & 0 & A. Possenti 2010, unpublished \\
\hline NGC 6544 & 3.0 & 38.8 & 93.5 & -1.40 & 108,000 & 0 & A. Possenti 2010, unpublished \\
\hline NGC 6541 & 7.5 & 9.6 & 42.2 & -1.81 & 572,000 & 0 & Lynch unpublished 2010 \\
\hline NGC 6558 & 7.4 & 63.3 & 32.1 & -1.32 & 98,400 & 0 & A. Possenti 2010, unpublished \\
\hline NGC 6584 & 13.5 & 54.8 & 24.3 & -1.50 & 303,000 & 0 & A. Possenti 2010, unpublished \\
\hline NGC 6624 & 7.9 & 16.0 & 35.3 & -0.44 & 257,000 & 2 & Lynch et al. 2011 \\
\hline M 28 & 5.5 & 4.1 & 63.8 & -1.32 & 551,000 & 0 & Lynch unpublished 2010 \\
\hline NGC 6642 & 8.1 & 54.8 & 30.7 & -1.26 & 109,000 & 0 & A. Possenti 2010, unpublished \\
\hline NGC 6652 & 10.0 & 77.5 & 37.5 & -0.81 & 109,000 & 0 & Possenti unpublished 2010 \\
\hline M 22 & 3.2 & 5.7 & 44.7 & -1.70 & 644,000 & 0 & Lynch et al. 2011 \\
\hline NGC 6681 & 9.0 & 77.5 & 39.3 & -1.62 & 179,000 & 0 & Possenti unpublished 2010 \\
\hline NGC 6712 & 6.9 & 6.7 & 27.7 & -1.02 & 257,000 & 0 & Lynch et al. 2011 \\
\hline NGC 6717 & 7.1 & 54.8 & 21.6 & -1.26 & 47,500 & 0 & Possenti unpublished 2010 \\
\hline NGC 6723 & 8.7 & 54.8 & 27.3 & -1.10 & 357,000 & 0 & Possenti unpublished 2010 \\
\hline NGC 6749 & 7.9 & 32 & 20.4 & -1.60 & 123,000 & 0 & Hessels et al. 2007 \\
\hline NGC 6752 & 4.0 & 31.5 & 32.9 & -1.54 & 317,000 & 0 & Possenti unpublished 2010 \\
\hline NGC 6760 & 7.4 & 37 & 40.1 & -0.40 & 357,000 & 0 & Hessels et al. 2007 \\
\hline NGC 6779 & 9.4 & 25 & 28.7 & -1.98 & 230,000 & 0 & Hessels et al. 2007 \\
\hline Pal 10 & 5.9 & 23 & 17.2 & -0.10 & 53,100 & 0 & Hessels et al. 2007 \\
\hline NGC 6809 & 5.4 & 77.5 & 19.7 & -1.94 & 269,000 & 0 & Possenti unpublished 2010 \\
\hline NGC 6838 & 4.0 & 19 & 16.7 & -0.78 & 43,000 & 0 & Hessels et al. 2007 \\
\hline NGC 6934 & 15.6 & 28 & 28.1 & -1.47 & 295,000 & 0 & Hessels et al. 2007 \\
\hline NGC 6981 & 17.0 & 6.0 & 16.6 & -1.42 & 168,000 & 0 & Lynch \& Ransom 2011 \\
\hline NGC 7006 & 41.2 & 19 & 19.8 & -1.52 & 303,000 & 0 & Hessels et al. 2007 \\
\hline NGC 7078 & 10.4 & 21 & 62.1 & -2.37 & $1,190,000$ & 0 & Hessels et al. 2007 \\
\hline NGC 7089 & 11.5 & 6.0 & 48.1 & -1.65 & 104,000 & 0 & Lynch \& Ransom 2011 \\
\hline NGC 7099 & 8.1 & 10.8 & 34.1 & -2.27 & 241,000 & 0 & Ransom et al. 2004 \\
\hline Pal 12 & 19.0 & 6.2 & 5.5 & -0.85 & 15,900 & 0 & Lynch \& Ransom 2011 \\
\hline Pal 13 & 26.0 & 21 & 3.5 & -1.88 & 6,500 & 0 & Hessels et al. 2007 \\
\hline
\end{tabular}

while for the two pulsars in NGC 6624, $n=2$ which leads to

$$
p(n \mid N, \theta)=\frac{N(N-1)}{2} \theta^{2}(1-\theta)^{N-2} .
$$

Having found the joint posterior distribution $p(N, \theta \mid n)$, we then marginalize over $\theta$ to get the posterior distribution for $N$ using the appropriate choice for the likelihood function (i.e., Equations (5), (6), or (7) depending on the value for $n$ in each case) and give the $95 \%$ percentile-based credible intervals for $N$ (i.e., the 0.025 and 0.975 percentiles) as well as the median in Table 4. Examples of these discrete probability density functions can be seen in Figure 2 for four GCs. 47 Tuc shows the typical shape of a discrete probability density function for a GC with no young pulsars.

\section{THE INTRINSIC POPULATION AND BIRTHRATES OF YOUNG GC PULSARS}

The results from the previous sections do not take into account the population of young pulsars whose emission beams do not intersect our line of sight or the population of young pulsars which escaped the gravitational potential of their parent GCs. Both of these issues are addressed in this section.

\subsection{Results for all GCs}

Retention fractions $\left(f_{R}\right)$, the fraction of pulsars which do not have a large enough birth velocity to escape the cluster's gravitational potential, are calculated for each GC. These $f_{R}$ are obtained by using the escape velocity of the GC and a velocity distribution function. Hobbs et al. (2005) showed that a Maxwellian distribution fits the Galactic population of pulsars well for many categories (all, young, recycled, etc.) of pulsars, hence we choose the velocity distribution function to have the form of a Maxwellian with a dispersion $\sigma_{v}$. Caution does need to be taken with this choice of velocity distribution function because no physical mechanism is presented in Hobbs et al. (2005) to explain this Maxwellian distribution and the low velocity end of this distribution is not well constrained due to the fact low velocity pulsar's proper motions are difficult to measure. Multiple values were chosen for $\sigma_{v}: 265 \mathrm{~km} \mathrm{~s}^{-1}$ from Hobbs et al. (2005), an intermediate value of 130, 50, 20, and $10 \mathrm{~km} \mathrm{~s}^{-1}$ as a lower value. Many separate values have been chosen because it is most likely that these young GC pulsars are formed from ECSs for which the accompanying natal kick may be 10 times smaller than in the case of core-collapse supernova (Ivanova et al. 2008; Kitaura et al. 2006). The escape velocities were taken from Gnedin et al. (2002) except for ESO 452 which came from Webbink (1985). The retention fractions are calculated by numerically integrating the Maxwellian 
Table 4

Binomial Analysis of Young Pulsars in Globular Clusters

\begin{tabular}{|c|c|c|c|c|c|c|c|c|c|c|}
\hline $\begin{array}{l}\text { Globular } \\
\text { Cluster }\end{array}$ & Median & $\begin{array}{c}2.5 \text { th } \\
\text { percentile }\end{array}$ & $\begin{array}{c}\text { 97.5th } \\
\text { percentile }\end{array}$ & $\begin{array}{c}\log \left(f_{R 1}\right) \\
50\left(\mathrm{~km} \mathrm{~s}^{-1}\right)\end{array}$ & $\begin{array}{c}\log \left(f_{R 2}\right) \\
20\left(\mathrm{~km} \mathrm{~s}^{-1}\right)\end{array}$ & $\begin{array}{c}\log \left(f_{R 3}\right) \\
10\left(\mathrm{~km} \mathrm{~s}^{-1}\right)\end{array}$ & $\begin{array}{c}\log (\mathcal{R}) f_{R 1} \\
\text { (pulsars century }{ }^{-1} \text { ) }\end{array}$ & $\begin{array}{c}\log (\mathcal{R}) f_{R 2} \\
\text { (pulsars century }{ }^{-1} \text { ) }\end{array}$ & $\begin{array}{c}\log (\mathcal{R}) f_{R 3} \\
\text { (pulsars century }^{-1} \text { ) }\end{array}$ & $\begin{array}{c}N_{\text {predicted }} \\
\text { Mass Model }\end{array}$ \\
\hline 47 Tuc & 16 & 0 & 88 & -0.39 & 0.00 & 0.00 & -3.04 & -3.43 & -3.43 & 1 \\
\hline NGC 288 & 3 & 0 & 17 & -2.30 & -1.16 & -0.42 & -1.85 & -3.00 & -3.74 & 0 \\
\hline NGC 1261 & 53 & 1 & 297 & -4.08 & -2.88 & -1.99 & 1.15 & -0.03 & -0.92 & 0 \\
\hline Pal 2 & 167 & 6 & 968 & -1.37 & -0.38 & -0.02 & -1.03 & -2.03 & -2.40 & 0 \\
\hline NGC 1851 & 21 & 0 & 115 & -0.66 & -0.03 & 0.00 & -2.65 & -3.28 & -3.32 & 0 \\
\hline NGC 2298 & 3 & 0 & 16 & -1.89 & -0.79 & -0.17 & -2.26 & -3.37 & -3.99 & 3 \\
\hline NGC 2808 & 24 & 0 & 133 & -0.34 & 0.00 & 0.00 & -2.92 & -3.26 & -3.26 & 0 \\
\hline E3 & 17 & 0 & 93 & -4.24 & -3.05 & -2.15 & 0.82 & -0.36 & -1.25 & 0 \\
\hline NGC 3201 & 6 & 0 & 36 & -1.66 & -0.60 & -0.08 & -2.19 & -3.26 & -3.77 & 0 \\
\hline NGC 4147 & 34 & 1 & 192 & -1.90 & -0.79 & -0.17 & -1.20 & -2.31 & -2.93 & 0 \\
\hline NGC 4372 & 9 & 0 & 49 & -1.70 & -0.62 & -0.09 & -1.99 & -3.06 & -3.59 & 0 \\
\hline NGC 4590 & 28 & 1 & 155 & -1.90 & -0.80 & -0.18 & -1.28 & -2.39 & -3.01 & 0 \\
\hline NGC 4833 & 16 & 0 & 87 & -1.21 & -0.27 & 0.00 & -2.22 & -3.16 & -3.43 & 0 \\
\hline NGC 5024 & 29 & 1 & 163 & -1.15 & -0.23 & 0.00 & -2.02 & -2.94 & -3.17 & 0 \\
\hline NGC 5053 & 29 & 1 & 162 & -2.83 & -1.65 & -0.82 & -0.35 & -1.52 & -2.35 & 0 \\
\hline NGC 5139 & 6 & 0 & 36 & -0.50 & 0.00 & 0.00 & -3.35 & -3.85 & -3.86 & 8 \\
\hline NGC 5272 & 10 & 0 & 57 & -1.03 & -0.16 & 0.00 & -2.61 & -3.47 & -3.64 & 1 \\
\hline NGC 5286 & 37 & 1 & 205 & -0.64 & -0.03 & 0.00 & -2.42 & -3.04 & -3.07 & 0 \\
\hline NGC 5466 & 27 & 0 & 149 & -2.74 & -1.57 & -0.75 & -0.46 & -1.63 & -2.45 & 0 \\
\hline Pal 5 & 101 & 3 & 575 & -4.16 & -2.96 & -2.07 & 1.51 & 0.32 & -0.56 & 0 \\
\hline NGC 5897 & 4 & 0 & 25 & -2.30 & -1.15 & -0.41 & -1.73 & -2.88 & -3.62 & 0 \\
\hline NGC 5904 & 7 & 0 & 39 & -0.75 & -0.05 & 0.00 & -3.04 & -3.73 & -3.80 & 2 \\
\hline NGC 5927 & 15 & 0 & 84 & -1.13 & -0.23 & 0.00 & -2.32 & -3.23 & -3.46 & 0 \\
\hline NGC 5946 & 29 & 1 & 164 & -1.49 & -0.46 & -0.03 & -1.68 & -2.71 & -3.13 & 0 \\
\hline NGC 5986 & 2 & 0 & 14 & -1.03 & -0.17 & 0.00 & -3.30 & -4.16 & -4.34 & 3 \\
\hline M 80 & 3 & 0 & 19 & -0.73 & -0.05 & 0.00 & -3.43 & -4.11 & -4.16 & 6 \\
\hline NGC 6121 & 3 & 0 & 20 & -1.13 & -0.22 & 0.00 & -3.03 & -3.94 & -4.16 & 0 \\
\hline ESO452 & 17 & 0 & 98 & -3.36 & -2.17 & -1.30 & -0.04 & -1.23 & -2.10 & 0 \\
\hline NGC 6144 & 20 & 0 & 113 & -2.23 & -1.09 & -0.36 & -1.10 & -2.24 & -2.97 & 0 \\
\hline NGC 6139 & 39 & 1 & 218 & -0.53 & -0.01 & 0.00 & -2.51 & -3.03 & -3.05 & 0 \\
\hline NGC 6171 & 10 & 0 & 59 & -1.50 & -0.47 & -0.04 & -2.13 & -3.16 & -3.60 & 0 \\
\hline NGC 6205 & 7 & 0 & 37 & -0.97 & -0.14 & 0.00 & -2.82 & -3.65 & -3.80 & 1 \\
\hline NGC 6218 & 6 & 0 & 35 & -1.34 & -0.36 & -0.01 & -2.51 & -3.50 & -3.84 & 0 \\
\hline NGC 6235 & 35 & 1 & 197 & -2.01 & -0.89 & -0.23 & -1.08 & -2.20 & -2.86 & 0 \\
\hline NGC 6254 & 6 & 0 & 36 & -1.30 & -0.33 & -0.01 & -2.55 & -3.53 & -3.85 & 0 \\
\hline Pal 15 & 878 & 30 & 5409 & -3.77 & -2.58 & -1.70 & 2.07 & 0.88 & 0.00 & 0 \\
\hline NGC 6266 & 5 & 0 & 29 & -0.13 & 0.00 & 0.00 & -3.80 & -3.94 & -3.94 & 3 \\
\hline NGC 6273 & 20 & 0 & 110 & -0.54 & -0.01 & 0.00 & -2.80 & -3.32 & -3.34 & 0 \\
\hline NGC 6284 & 69 & 2 & 391 & -1.34 & -0.35 & -0.01 & -1.46 & -2.44 & -2.78 & 0 \\
\hline NGC 6287 & 33 & 1 & 185 & -1.26 & -0.30 & 0.00 & -1.85 & -2.81 & -3.11 & 0 \\
\hline NGC 6293 & 23 & 0 & 130 & -0.90 & -0.10 & 0.00 & -2.38 & -3.16 & -3.28 & 0 \\
\hline NGC 6304 & 9 & 0 & 51 & -0.99 & -0.15 & 0.00 & -2.69 & -3.53 & -3.69 & 0 \\
\hline M 92 & 2 & 0 & 13 & -0.76 & -0.06 & 0.00 & -3.57 & -4.28 & -4.34 & 2 \\
\hline NGC 6325 & 15 & 0 & 86 & -0.87 & -0.10 & 0.00 & -2.58 & -3.36 & -3.46 & 0 \\
\hline NGC 6333 & 16 & 0 & 88 & -1.01 & -0.16 & 0.00 & -2.42 & -3.27 & -3.43 & 0 \\
\hline NGC 6342 & 288 & 40 & 1047 & -1.61 & -0.56 & -0.06 & -0.56 & -1.62 & -2.11 & 0 \\
\hline NGC 6355 & 31 & 1 & 176 & -0.93 & -0.12 & 0.00 & -2.21 & -3.02 & -3.15 & 0 \\
\hline Liller 1 & 3 & 0 & 21 & -0.91 & -0.11 & 0.00 & -3.25 & -4.05 & -4.16 & 0 \\
\hline Ter 1 & 16 & 0 & 90 & -3.06 & -1.88 & -1.03 & -0.36 & -1.55 & -2.40 & 0 \\
\hline NGC 6388 & 25 & 0 & 142 & -0.04 & 0.00 & 0.00 & -3.20 & -3.24 & -3.24 & 1 \\
\hline NGC 6402 & 22 & 0 & 124 & -0.97 & -0.14 & 0.00 & -2.32 & -3.16 & -3.30 & 0 \\
\hline NGC 6401 & 43 & 1 & 244 & -0.99 & -0.15 & 0.00 & -2.01 & -2.85 & -3.00 & 0 \\
\hline NGC 6397 & 1 & 0 & 8 & -0.73 & -0.05 & 0.00 & -3.90 & -4.59 & -4.64 & 1 \\
\hline Pal 6 & 1 & 0 & 10 & -1.36 & -0.37 & -0.02 & -3.27 & -4.26 & -4.62 & 2 \\
\hline NGC 6426 & 55 & 1 & 308 & -2.18 & -1.04 & -0.33 & -0.72 & -1.85 & -2.56 & 0 \\
\hline Ter 5 & 3 & 0 & 18 & -0.69 & -0.03 & 0.00 & -3.47 & -4.12 & -4.16 & 2 \\
\hline NGC 6440 & 10 & 2 & 34 & -0.22 & 0.00 & 0.00 & -3.41 & -3.64 & -3.64 & 3 \\
\hline NGC 6441 & 8 & 0 & 45 & -0.12 & 0.00 & 0.00 & -3.62 & -3.74 & -3.74 & 3 \\
\hline Ter 6 & 2 & 0 & 13 & -0.99 & -0.15 & 0.00 & -3.34 & -4.19 & -4.34 & 1 \\
\hline NGC 6453 & 56 & 2 & 316 & -1.64 & -0.58 & -0.07 & -1.24 & -2.30 & -2.81 & 0 \\
\hline UKS 1 & 15 & 0 & 86 & -1.49 & -0.46 & -0.03 & -1.97 & -3.00 & -3.42 & 0 \\
\hline NGC 6496 & 34 & 1 & 189 & -1.80 & -0.71 & -0.13 & -1.30 & -2.39 & -2.97 & 0 \\
\hline Ter 9 & 4 & 0 & 25 & -2.70 & -1.53 & -0.72 & -1.33 & -2.50 & -3.32 & 0 \\
\hline NGC 6517 & 2 & 0 & 13 & -0.24 & 0.00 & 0.00 & -4.10 & -4.34 & -4.34 & 3 \\
\hline NGC 6522 & 15 & 0 & 84 & -0.88 & -0.10 & 0.00 & -2.58 & -3.36 & -3.46 & 0 \\
\hline
\end{tabular}


Table 4

(Continued)

\begin{tabular}{|c|c|c|c|c|c|c|c|c|c|c|}
\hline $\begin{array}{l}\text { Globular } \\
\text { Cluster }\end{array}$ & Median & $\begin{array}{c}2.5 \text { th } \\
\text { percentile }\end{array}$ & $\begin{array}{c}97.5 \text { th } \\
\text { percentile }\end{array}$ & $\begin{array}{c}\log \left(f_{R 1}\right) \\
50\left(\mathrm{~km} \mathrm{~s}^{-1}\right)\end{array}$ & $\begin{array}{c}\log \left(f_{R 2}\right) \\
20\left(\mathrm{~km} \mathrm{~s}^{-1}\right)\end{array}$ & $\begin{array}{c}\log \left(f_{R 3}\right) \\
10\left(\mathrm{~km} \mathrm{~s}^{-1}\right)\end{array}$ & $\begin{array}{c}\log (\mathcal{R}) f_{R 1} \\
\text { (pulsars century }^{-1} \text { ) }\end{array}$ & $\begin{array}{c}\log (\mathcal{R}) f_{R 2} \\
\text { (pulsars century }^{-1} \text { ) }\end{array}$ & $\begin{array}{c}\log (\mathcal{R}) f_{R 3} \\
\text { (pulsars century }^{-1} \text { ) }\end{array}$ & $\begin{array}{c}N_{\text {predicted }} \\
\text { Mass Model }\end{array}$ \\
\hline NGC 6528 & 16 & 0 & 88 & -1.44 & -0.42 & -0.03 & -1.99 & -3.01 & -3.40 & 0 \\
\hline NGC 6535 & 9 & 0 & 50 & -2.67 & -1.50 & -0.70 & -1.01 & -2.17 & -2.98 & 0 \\
\hline NGC 6539 & 13 & 0 & 74 & -1.07 & -0.19 & 0.00 & -2.45 & -3.33 & -3.53 & 0 \\
\hline NGC 6540 & 10 & 0 & 57 & -1.38 & -0.38 & -0.02 & -2.25 & -3.25 & -3.62 & 0 \\
\hline NGC 6544 & 2 & 0 & 13 & -0.16 & 0.00 & 0.00 & -4.17 & -4.34 & -4.34 & 0 \\
\hline NGC 6541 & 3 & 0 & 18 & -0.88 & -0.10 & 0.00 & -3.28 & -4.06 & -4.16 & 3 \\
\hline NGC 6558 & 16 & 0 & 90 & -1.20 & -0.26 & 0.00 & -2.23 & -3.16 & -3.43 & 0 \\
\hline NGC 6584 & 51 & 1 & 287 & -1.54 & -0.50 & -0.05 & -1.39 & -2.43 & -2.88 & 0 \\
\hline NGC 6624 & 21 & 5 & 58 & -1.09 & -0.20 & 0.00 & -2.23 & -3.11 & -3.32 & 0 \\
\hline M 28 & 1 & 0 & 7 & -0.45 & 0.00 & 0.00 & -4.18 & -4.63 & -4.64 & 10 \\
\hline NGC 6642 & 17 & 0 & 93 & -1.25 & -0.30 & 0.00 & -2.15 & -3.11 & -3.40 & 0 \\
\hline NGC 6652 & 38 & 1 & 213 & -1.02 & -0.16 & 0.00 & -2.04 & -2.90 & -3.06 & 0 \\
\hline M 22 & 0 & 0 & 4 & -0.82 & -0.07 & 0.00 & $-1.74^{\mathrm{a}}$ & $-2.66^{\mathrm{a}}$ & $-4.64^{\mathrm{a}}$ & 0 \\
\hline NGC 6681 & 30 & 1 & 168 & -0.96 & -0.13 & 0.00 & -2.20 & -3.02 & -3.16 & 0 \\
\hline NGC 6712 & 2 & 0 & 12 & -1.38 & -0.38 & -0.02 & -2.96 & -3.95 & -4.32 & 1 \\
\hline NGC 6717 & 13 & 0 & 72 & -1.69 & -0.62 & -0.09 & -1.83 & -2.90 & -3.43 & 0 \\
\hline NGC 6723 & 19 & 0 & 108 & -1.40 & -0.39 & -0.02 & -1.96 & -2.96 & -3.33 & 0 \\
\hline NGC 6749 & 9 & 0 & 53 & -1.76 & -0.67 & -0.12 & -1.92 & -3.00 & -3.56 & 0 \\
\hline NGC 6752 & 3 & 0 & 17 & -1.17 & -0.24 & 0.00 & -2.99 & -3.91 & -4.16 & 1 \\
\hline NGC 6760 & 9 & 0 & 53 & -0.94 & -0.13 & 0.00 & -2.74 & -3.56 & -3.69 & 0 \\
\hline NGC 6779 & 10 & 0 & 58 & -1.33 & -0.35 & -0.01 & -2.30 & -3.28 & -3.62 & 0 \\
\hline Pal 10 & 4 & 0 & 24 & -1.98 & -0.86 & -0.21 & -2.06 & -3.17 & -3.82 & 0 \\
\hline NGC 6809 & 10 & 0 & 59 & -1.80 & -0.71 & -0.13 & -1.83 & -2.92 & -3.50 & 0 \\
\hline NGC 6838 & 2 & 0 & 12 & -2.01 & -0.90 & -0.23 & -2.32 & -3.44 & -4.10 & 0 \\
\hline NGC 6934 & 33 & 1 & 184 & -1.36 & -0.37 & -0.01 & -1.75 & -2.75 & -3.10 & 0 \\
\hline NGC 6981 & 8 & 0 & 46 & -2.02 & -0.90 & -0.24 & -1.71 & -2.83 & -3.49 & 0 \\
\hline NGC 7006 & 237 & 8 & 1384 & -1.80 & -0.71 & -0.13 & -0.46 & -1.55 & -2.13 & 0 \\
\hline NGC 7078 & 11 & 0 & 60 & -0.48 & 0.00 & 0.00 & -3.11 & -3.59 & -3.60 & 2 \\
\hline NGC 7089 & 4 & 0 & 24 & -0.74 & -0.05 & 0.00 & -3.30 & -3.98 & -4.04 & 3 \\
\hline NGC 7099 & 4 & 0 & 22 & -1.13 & -0.22 & 0.00 & -2.90 & -3.81 & -4.03 & 1 \\
\hline Pal 12 & 10 & 0 & 59 & -3.45 & -2.26 & -1.39 & -0.18 & -1.37 & -2.25 & 0 \\
\hline Pal 13 & 79 & 2 & 446 & -4.04 & -2.85 & -1.96 & 1.29 & 0.10 & -0.78 & 0 \\
\hline
\end{tabular}

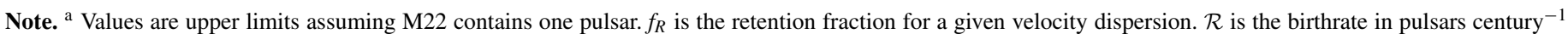
for the median value. Birthrates are upper limits for a given GC except for those with detected young pulsars.

velocity distribution function from zero to the escape velocity of the GCs.

Each retention fraction was used to calculate a theoretical upper limit for the number of young pulsars produced for every GC

$$
N_{\text {created }}=\frac{N}{f_{R} f_{\text {beam }}},
$$

where $N$ represents the number of pulsars predicted by the binomial method, i.e., the median in Table 4 , and $f_{\text {beam }}$ is the beaming fraction for pulsars. The value taken for the pulsar beaming fraction is 0.1 (Tauris \& Manchester 1998). This value represents an upper limit on the number of pulsars created in a particular GC. The number of pulsars created in a GC is then divided by the average lifetime of a young pulsar (43 Myr) to obtain upper limits on the birthrates $(\mathcal{R})$ for each GC. An average lifetime of $43 \mathrm{Myr}$ is derived by taking the total number of radio-loud pulsars $(1,200,000)$ and dividing it by the pulsar birthrate (2.8 pulsars century $\left.{ }^{-1}\right)$ in FK06. All results from the binomial analysis are contained within Table 4.

One GC, M22, has a median value of zero and no young pulsars are expected to be contained within it. This results in a birthrate of zero for M22. To calculate a birthrate for this GC, it is assumed that the cluster contains one pulsar and an upper limit is constructed using this assumption.

\subsection{Metal-rich GCs}

Up to this point, the analysis presented uses flux density limits and luminosity models as the only considerations for observable pulsars contained within the cluster. Lyne et al. (1996) note that the young pulsars appear in metal-rich GCs and this trend has persisted despite 15 years of intense searches of most of the cluster population. In this section, metallicity will also be added as a consideration. For the following discussion, Terzan 5 will be excluded due to the possibility that it is not a true GC but a merger of two astrophysical objects that are bound in the Galactic halo (Ferraro et al. 2009). Also excluded will be B1718-19 in NGC 6342 due to the uncertainty about its membership to its cluster (see Section 2).

The GCs NGC 6440 and NGC 6624 have metallicities that are greater than the 90 th percentile of metal-rich clusters. These two clusters are two of the three highest metallicity clusters that contain either young or old MSPs. The probability of selecting two GCs in the top three of a ranked list from a sample of 25 GCs is $0.92 \%$. If NGC 6342 is also included in this sample then we have three of the top five highest metallicity clusters with a probability of selecting three of the top five of $0.38 \%$. The inclusion of Terzan 5 (the highest metallicity cluster with any known pulsars) in both of these previous scenarios, changes the probability values from $0.92 \%$ to $1.71 \%$ and $0.38 \%$ to $0.68 \%$. A conjecture can be proposed that metal-poor clusters have been 

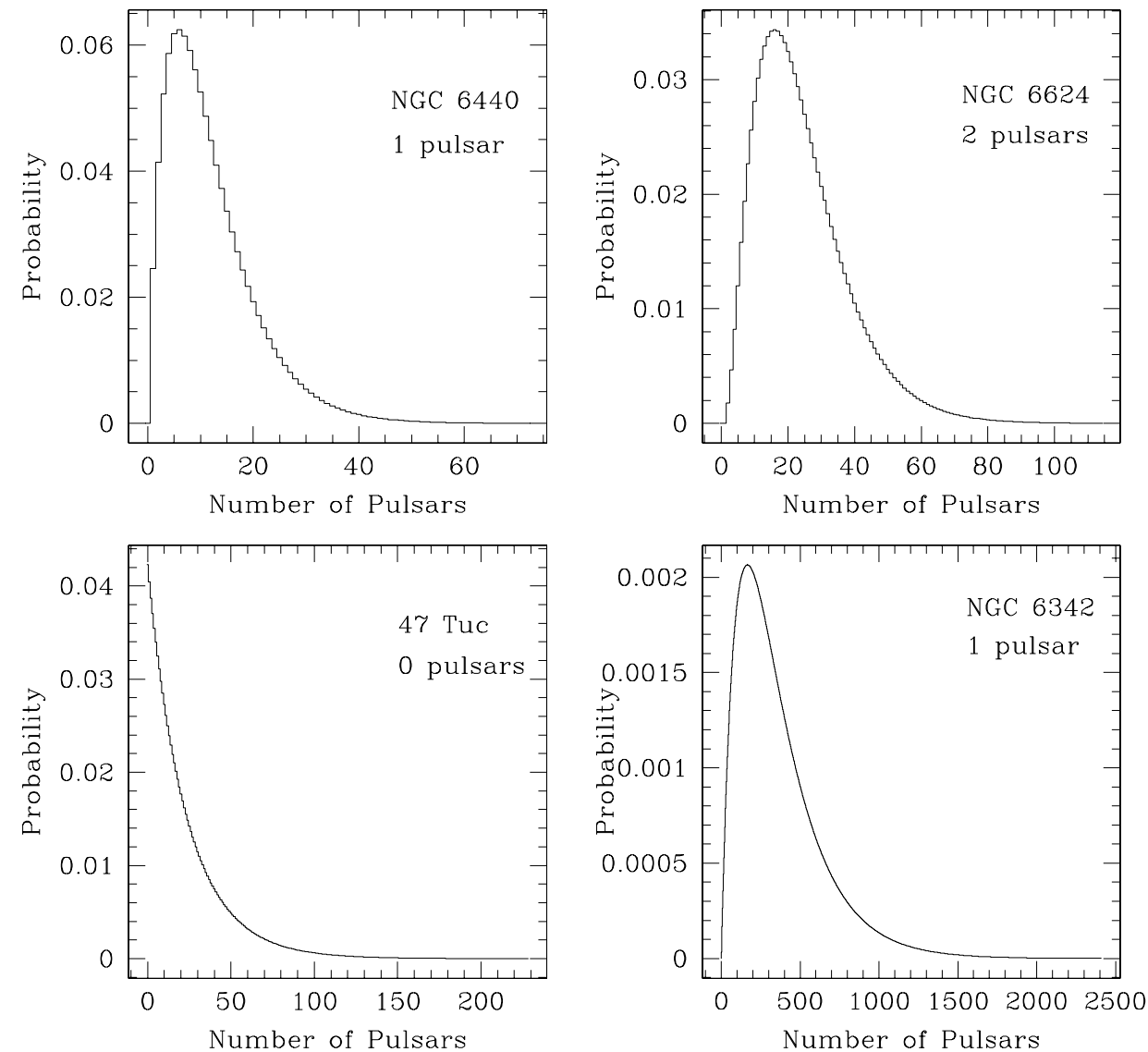

Figure 2. Examples of discrete posterior probability density functions for the number of potentially observable pulsars in GCs using the Bayesian analysis.

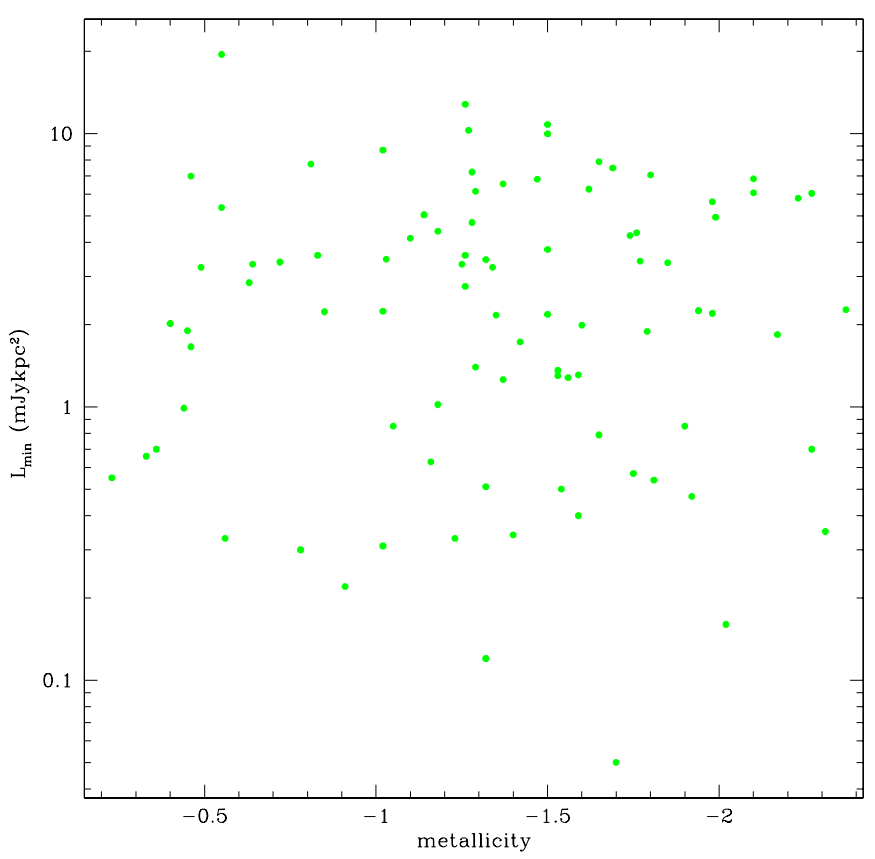

Figure 3. $1400 \mathrm{MHz}$ luminosity survey limits as a function of metallicity with six GCs excluded with distances greater than $20 \mathrm{kpc}$. The luminosities are randomly distributed and show no bias against low- or high-metallicity GCs.

(A color version of this figure is available in the online journal.)

selected against for the purpose of surveying GCs due to the belief that metal-rich GCs contain more pulsars overall. Figure 3 shows a plot of metallicity versus the survey luminosity limit,

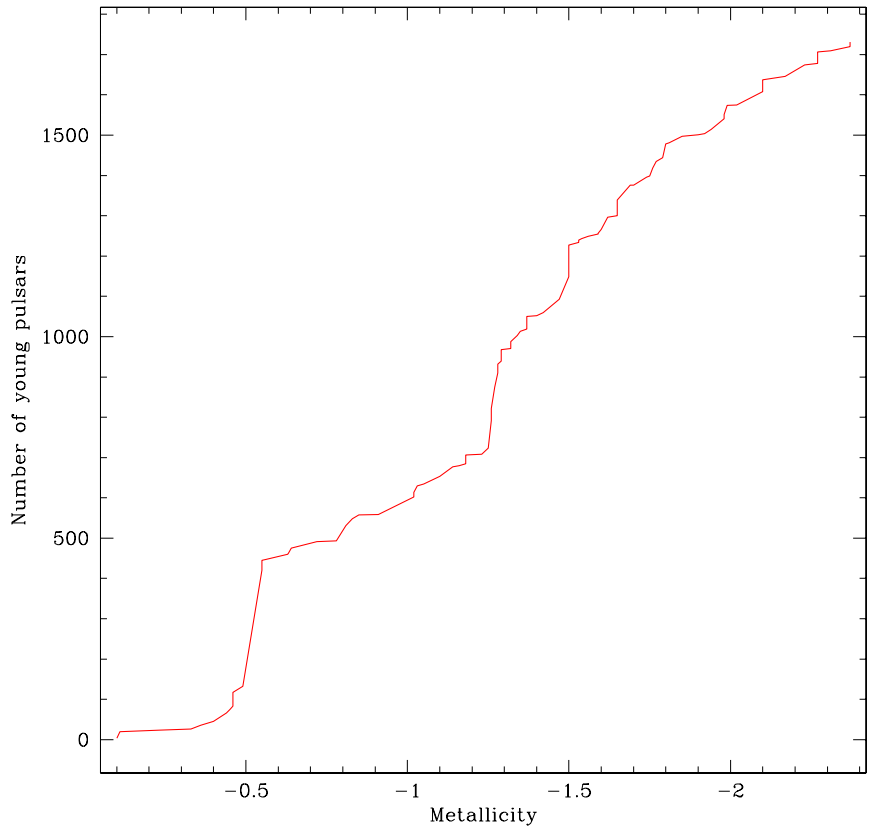

Figure 4. CDF of young pulsars predicted by the binomial analysis as a function of metallicity.

(A color version of this figure is available in the online journal.)

indicating a random distribution with no bias toward either lowor high-metallicity clusters.

Figure 4 shows the empirical cumulative distribution function (CDF) of the number of predicted young pulsars with the 
binomial method versus metallicity. GCs with distances greater than $20 \mathrm{kpc}$ are excluded from this CDF because their large distances produce high luminosity limits and in turn creates jumps in the CDF. The cause of these jumps seen in Figure 4 is due to high survey limits on specific GCs. The first large jump in the CDF at a metallicity of $\sim-0.55$ is due to NGC 6342 , the GC with the highest survey luminosity limit.

The GCs with the most known pulsars, Ter 5 and 47 Tuc, have high metallicities and have been used in many simulations. One of these presented in Ivanova et al. (2008) attempts to model the number of young/high magnetic field pulsars in these clusters. In these simulations, three and two young pulsars are predicted to exist in the cores of Ter 5 and 47 Tuc, respectively, with the most likely creation scenario being the merger of two stars. If beaming and luminosity limits from Table 3 are taken into account, the chances of seeing those 2-3 pulsars are extremely small.

\section{DISCUSSION}

\subsection{Flux Luminosity Limits}

The results of the binomial analysis depend greatly on the luminosity limits provided from searching these GCs. One can see by comparing the discrete probability density functions of NGC 6342 and NGC 6440 seen in Figure 2, that the range of values that each function covers differs greatly. This is a direct result of NGC 6342's flux luminosity limit being much greater than that of NGC 6440. Thus, clusters with high flux luminosity limits do not significantly constrain the population/birthrates of these GCs.

Another factor not mentioned is the influence of RFI on searching GCs. The relative impact of RFI on long-period pulsars is much more severe than for MSPs. There is a lot more long-period RFI, and the dispersion discrimination between RFI and long-period pulsars is not nearly as great as for MSPs. Therefore, this creates a bias against finding such pulsars because they may be ignored assuming they are RFI which is hard to quantify and fully account for.

\subsection{Birthrates in GCs}

For most GCs, the birthrates found are only upper limits with the exception of NGC 6342, NGC 6440, and NGC 6624. Empirical CDFs of these values can be seen in Figure 5 for the 97 GCs surveyed. The upper limits on birthrates for velocity dispersions of $265,130,50,20$, and $10 \mathrm{~km} \mathrm{~s}^{-1}$ are 3568,422 , $24.8,1.67$, and 0.25 pulsars century ${ }^{-1}$, respectively. The higher birthrates obtained from the higher velocity dispersions is an effect of needing to produce more pulsars to get enough pulsars at the low velocity end of the Maxwellian distribution. These values for birthrates provided in this work are much higher than the predicted birth of 2.8 pulsars century ${ }^{-1}$ for the Galaxy as a whole for larger velocity dispersions (Faucher-Giguère \& Kaspi 2006). The impact on the Galactic population from pulsars escaping GCs will be discussed elsewhere (R. S. Lynch et al. 2011 , in preparation). For the purposes of this paper, these very high implied birthrates suggest that a very different formation process for young pulsars is occurring in GCs, as well as the possibility that some GCs do not produce young pulsars at all. The major difference in the formation scenario appears to be the lower velocity dispersion at birth.

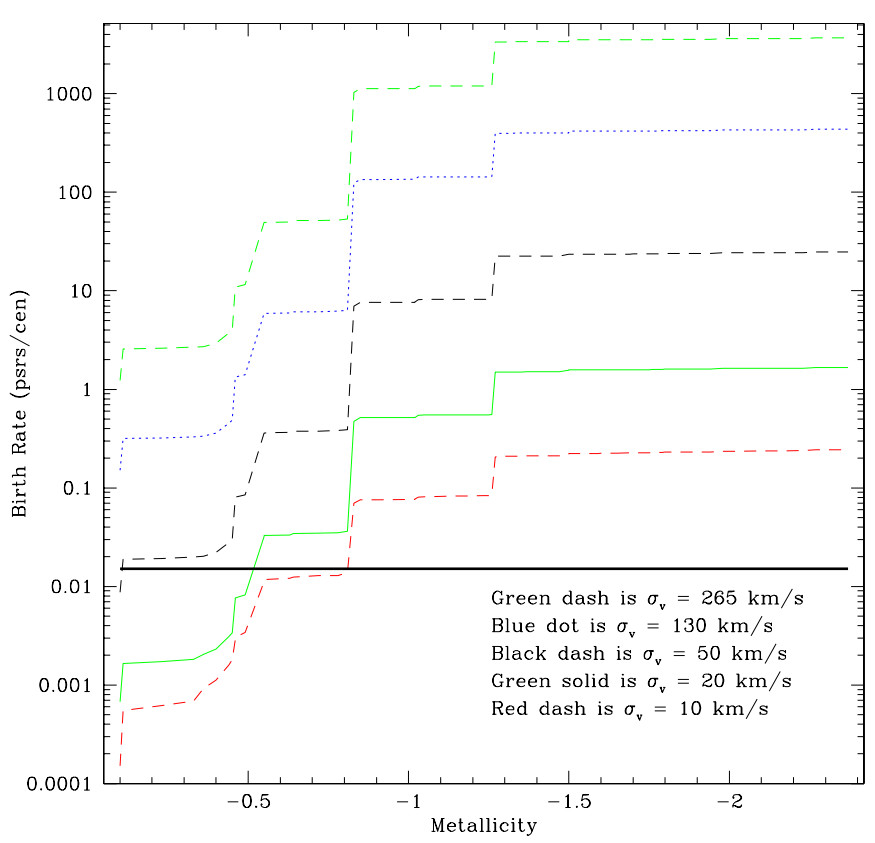

Figure 5. CDF of birthrate versus metallicity.

(A color version of this figure is available in the online journal.)

\subsection{Role of Cluster Metallicity}

In Section 5.2, evidence is presented that young pulsars are only present in higher metallicity GCs. Another way to highlight this is to use a simple population model with the GC's mass as the only variable. A pulsar to mass ratio (PMR) is derived using the results of the binomial analysis for NGC 6440 and NGC 6624, each having 10 and 21 pulsars predicted, respectively. We do not include the GC NGC 6342 when creating this model because of the uncertain cluster membership of B1718-19 (see Section 2). With NGC 6440's mass of $811,000 M_{\odot}$ and NGC 6624's mass of 257,000 $M_{\odot}$, a PMR of 31 pulsars per $1,068,000 M_{\odot}$ is obtained. Using the PMR, each of the $97 \mathrm{GCs}$ are revisited and an intrinsic population is predicted for each GC by multiplying the PMR by the GC mass. Using the flux density limits in Table 3, the observable population is drawn from the Faucher-Giguère \& Kaspi (2006) luminosity model. The results of this simulation can be seen in a histogram in Figure 6 and Table 4. Seventy-four percent of the GCs are predicted to have no observable young pulsars using this model. However, for the remaining GCs, a total of 67 young pulsars should be observable which disagrees with the current population of three by over an order of magnitude. These results show that mass is not a single determining factor in a GC containing a young pulsar.

Based on the observed population of young pulsars in the higher metallicity GCs, it may be suggested that lower metallicity GCs may not produce any young pulsars. Figure 5 may be used to predict birthrates as a function of metallicity. Given that no young pulsars are observed with metallicities below -0.6 , this value is adopted as a cutoff value. In this case, the population of young pulsars in GCs with $\log [\mathrm{Fe} / \mathrm{H}]>$ -0.6 is $447_{-399}^{+1420}$, at $95 \%$ confidence level. This implies an upper limit on the birthrate for GCs with $\log [\mathrm{Fe} / \mathrm{H}]>-0.6$ of $0.012_{-0.010}^{+0.037}$ pulsars century ${ }^{-1}$.

\subsection{Formation Scenarios}

A few possible situations need to be examined that could explain this current population of normal pulsars in GCs. 


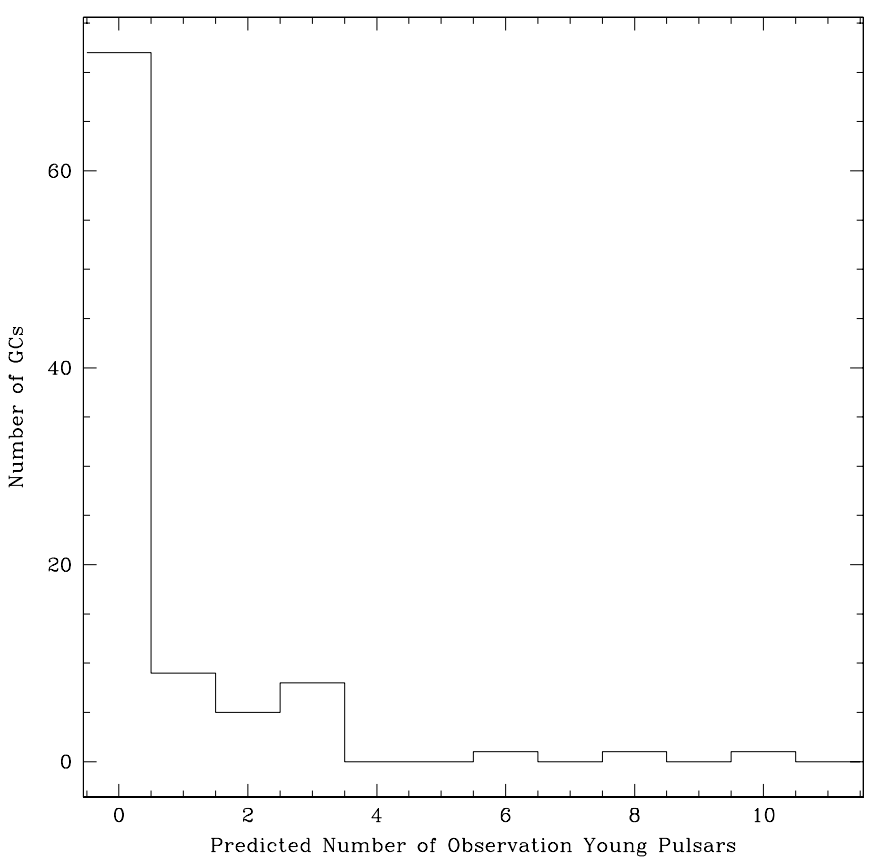

Figure 6. Histogram of number of predicted observable young pulsars per GC using a model only dependent on the mass of the GC.

\subsubsection{Blue Stragglers}

Blue stragglers are the product of the merger of two or possibly three $\sim 1 M_{\odot}$ main-sequence stars (Leonard 1989) or binary accretion of a binary companion(s) (McCrea 1964). They cannot be the progenitors of the young pulsars because they are not massive enough to core collapse.

\subsubsection{Electron Capture Supernovae}

The creation of these normal pulsars from an ECS of oxygen-neon-magnesium $(\mathrm{ONeMg})$ white dwarfs is another possible avenue. There are three main types of ECSs: accretion-induced collapse (AIC), evolution-induced collapse, and merger-induced collapse, of which AIC is the most common (Ivanova et al. 2008). From conservation of magnetic flux, a five order of magnitude change in surface magnetic field is available from an AIC of a white dwarf into a neutron star. This would require a white dwarf to have at least $10^{7} \mathrm{G}$ magnetic field to produce the magnetic field of the young pulsar with the largest magnetic field contained within a GC. White dwarfs have been observed with magnetic field above $2 \times 10^{6} \mathrm{G}$ and could account up to $10 \%$ of the white dwarf population (Liebert et al. 2003). ECSs could be an avenue for the creation of young pulsars. Not all white dwarfs that pass the Chandrasekhar limit will collapse via ECS, some will be Type Ia supernova.

ECSs provide two qualities that are needed to explain the presence of young pulsars in GCs. The first is the low velocity dispersion which is needed to keep these young pulsars in their host GCs. The initial formation of the white dwarf results in a small velocity kick of a few $\mathrm{km} \mathrm{s}^{-1}$ from either stellar winds or an asymmetric kick during the helium flash (Fregeau et al. 2009). The energetics of the ECS of the white dwarf would produce a small velocity kick (Dessart et al. 2006) and, combined with the velocity kick from previous stages of evolution, would provide a total velocity that is small and would allow most neutron stars created via ECS to be retained by the GC. The second is the higher metallicity of GCs which host young pulsars. An ECS ejects a few $0.001 M_{\odot}$ worth of mass, of which $\sim 25 \%$ is ${ }^{56} \mathrm{Ni}$
(Dessart et al. 2006). ${ }^{56} \mathrm{Ni}$ decays into ${ }^{56} \mathrm{Co}$ with a half-life of $\simeq 6$ days and then ${ }^{56} \mathrm{Co}$ decays into ${ }^{56} \mathrm{Fe}$ with a half-life of $\simeq 77$ days. This provides a source of iron creating the higher metallicity for clusters in which all known young pulsars have been detected.

A simple statistical analysis can give an order of magnitude estimate of the possible population of heavy mass (1.0-1.4 $\left.M_{\odot}\right)$ white dwarfs available for ECSs. It is taken that all GCs have the same initial mass function (IMF) and the differences seen between current GC's mass functions are due to the dynamical history of the cluster (Paust et al. 2010). The IMF is a multipart power law presented in Equation (4) of Kroupa (2002) and with $\alpha_{3}=-2.7$ from Scalo (1986). Each GC is assumed to have the same age of 12.4 Gyr for purposes of predicting an initial mass of the GC (Krauss \& Chaboyer 2001). An initial mass is obtained for each GC by using the mass lost fractions presented in Kroupa (2002) for $\alpha_{3}=-2.7$. The number of mainsequence stars available for heavy white dwarfs (mass range of 6.0-8.0 $M_{\odot}$ ) are calculated for each GC with a total of $\sim 150,000$ white dwarfs formed in these 97 GCs. The use of values for $\alpha_{3}$ greater than -2.7 serves only to decrease the numbers of stars in this mass range while choosing the Salpeter index of $\alpha_{3}=$ -2.35 only increases the number of stars available by a factor of two (Salpeter 1955). Assuming an interaction age of $10^{9} \mathrm{yr}$ and that each heavy white dwarf creates one ECS, an occurrence rate of $1.5 \times 10^{-2}$ ECSs century $^{-1}$ is obtained and is shown on Figure 5 as a solid horizontal black line.

This predicted ECS rate is an order of magnitude less than the birthrates of pulsars for the lowest velocity dispersion. A few factors could bring these values into closer agreement. A decrease in the interaction age would increase the ECS rate. This is a very plausible possibility because there is an accumulation of ECSs at more recent times due to dynamical evolution (it may take a long time for a given capture to form an accreting binary) and individual evolution (it may take several Gyrs to accrete enough mass to cause an ECS). Lower luminosity limits without the detection of more young pulsars would decrease the young pulsar birthrate and bring the two rates closer to agreement. A detailed $N$-body simulation should be used to refine the ECS rate but this is beyond the scope of this work. It is possible that ECSs have only occurred in higher metallicity cluster and not all GCs should be considered in calculating the birthrate of young pulsars. The work presented here suggests that ECS is the most likely creation scenario for young pulsars in GCs.

\subsubsection{Galactic Bulge Pulsars}

One other possibility is that these young pulsars are part of the Galactic field population and are captured by their host GC. The time it would take to travel the distances to the GCs and the time it would take for these pulsars to relax into the core of their host GC have been proposed as evidence against this method. One key fact is neglected here; these are all bulge GCs that contain this population of young pulsars. Their distances from the Galactic center range from 1.2 to $1.7 \mathrm{kpc}$ and the radius of the Galactic bulge is $\sim 1.5 \mathrm{kpc}$. The time for a pulsar to travel from the outer $0.5 \mathrm{kpc}$ of the Galactic bulge would be short $\left(\sim 10^{6} \mathrm{yr}\right)$ even for a moderate birth velocity kick and the core relaxation time for each $\mathrm{GC}$ is an order of magnitude less than the characteristic age of the pulsars that hosts them allowing enough time for them to settle into the core which is where three of the four pulsars are found. The fourth, PSR B1718-19, has a characteristic age about equal to NGC 6342's relaxation time and this could explain why PSR B1718-19 is not located 
in the host GC's core. This could also explain why these young pulsars are toward the higher end of the age distribution for nonrecycled pulsars. The metallicity of the Galactic center is higher than that of the Galactic disk and stars migrating from the bulge to bulge GCs could explain why GCs with young pulsars have higher metallicities compared to the overall GC population.

The population of bulge pulsars is relatively unexplored. Outside of Galactic center surveys (Deneva et al. 2009; Johnston et al. 2006; Klein et al. 2004; Kramer et al. 2000) and the surveys of bulge GCs (Table 3), the population is unknown. The most sensitive published survey to explore this region is the Parkes Multi-beam Survey (Manchester et al. 2001). For pulsars with a period of 100-1000 ms, the survey has a limiting flux of $0.16 \mathrm{mJy}$ and this translates into a $L_{\min }$ of $7.8 \mathrm{mJy} \mathrm{kpc}^{2}$ for a distance of $7.0 \mathrm{kpc}$ (front edge of bulge) and $12 \mathrm{mJy} \mathrm{kpc}^{2}$ for a distance of $8.6 \mathrm{kpc}$ (top and bottom of bulge directly above and below Galactic center). These $L_{\text {min }}$ values represent the upper edge of the pulsar luminosity function presented in FK06 and show that the Galactic bulge has not been surveyed well enough to constrain its pulsar population especially considering DM smearing and scattering would further hinder the detection of these pulsars.

Another chance for pulsars to be captured will occur when the GC passes through the plane of the Galaxy as it orbits the Galactic center. The orbital timescales for GCs around the galaxy are hundreds of Myrs and this is longer than the $43 \mathrm{Myr}$ average lifetime of a normal pulsar. This means that any pulsars picked up would have to have been picked up on the most recent pass of the GC through the Galactic disk. Figure 1 shows a small population of pulsars that would live long enough to be found in a GC if capture occurred due to this scenario.

The plausibility of star capture by a GC is discussed in Mieske \& Baumgardt (2007; henceforth MB07) and their arguments will be applied here in the following discussions. Table 2 lists all GCs within $1.5 \mathrm{kpc}$ of the Galactic center and their characteristics relevant to the MB07 analysis. MB07 found that the capture probability decreases with increasing number of cluster particles $\left(N_{\mathrm{GC}}\right)$ and decreases with increasing initial velocity over cluster velocity dispersion ratio $\left(\frac{V_{\text {int }}}{\sigma_{\mathrm{GC}}}\right)$. Assuming an average particle mass of $0.5 M_{\odot}$, the range of clusters reviewed matches with the range of Galactic bulge clusters, however the range of $\frac{V_{\text {int }}}{\sigma_{\mathrm{GC}}}$ do not. GC velocity dispersions range from $\sim 1.0$ to $19 \mathrm{~km} \mathrm{~s}^{-1}$, which are an order of magnitude less than typical pulsar velocities in previous studies (see Hobbs et al. 2005, for a recent study). The closest scenario of $\frac{V_{\text {int }}}{\sigma_{\mathrm{GC}}}$ equal to unity will be the only one considered from here.

To obtain a comparison of capture rates between pulsars and MB07, a few other considerations need to be put into place. Using the FK06 model for the Galactic pulsar population, the mass density of pulsars is found to be $8.2 \times 10^{-6} M_{\odot} \mathrm{pc}^{-3}$ for the Galactic bulge assuming a uniform pulsar per mass distribution throughout the Galaxy. This value is five orders of magnitude less than the value of $0.25 M_{\odot} \mathrm{pc}^{-3}$ used in MB07. Even if the entire pulsar population were placed inside the Galactic bulge, this would increase the mass density by only an order of magnitude. The resulting differences in mass density would produce significantly lower values for the rates found by MB07. The value of $265 \mathrm{~km} \mathrm{~s}^{-1}$ will be adopted as the velocity dispersion for field pulsars $\left(\sigma_{\text {field }}\right)$. For a $\sigma_{\text {field }}$ of $200 \mathrm{~km} \mathrm{~s}^{-1}$ MB07 concludes that no stars will be captured in any mass cluster within a Hubble time. If the additional constraints for pulsars of lower capture probability due to higher initial velocities and lower capture rates due to lower mass density are included, the conclusion can be drawn that no pulsars are likely to be captured by a GC. Imposing further constraints of beaming, luminosity limits, and finite radio-loud lifetimes for pulsars would further hinder the detection of a pulsar if one were to be captured by a GC. We therefore rule out this possibility as an origin for the young pulsars in GCs.

\subsection{Suggested Future Work}

All the work presented here only provides a statistical study of the young pulsar population in GCs and neglects the dynamics and history of each GC. Clearly $N$-body simulations similar to those presented in Ivanova et al. (2008) of the GCs and their possible interactions with the Galactic stellar and poststellar populations would place better constraints on the values presented here and would further the understanding of GC's dynamics and evolutionary history.

To improve upon the observational constraints used in this work, it is clearly desirable to search all GCs as deeply as possible using existing facilities. We highlight here some GCs of particular interest.

There are a few exceptions, most notably NGC 6342 which has the highest minimum detectable flux density of any of the 97 GCs and has not to our knowledge been surveyed since the discovery of B1718-19 by Lyne et al. (1993). A new search with the currently available telescopes could reduce $S_{\min }$ by a factor of 10 and provide evidence for or against PSR B1718-19 association with NGC 6342 (Bailes et al. 2005; Freire 2005).

Another high-metallicity bulge GC to be searched is NGC 6637 (M 69). This cluster, at a distance of $8.8 \mathrm{kpc}$, also has a high two-body encounter rate, $\Gamma$. This parameter is often used to assess the plausibility of pulsar content in GCs (see, e.g., Hui et al. 2010).

Most of the GCs presented in this work have been searched to the sensitivity limit of the telescope for which they are visible. The next generation of radio telescopes will be needed to present better constraints on this work. The Square Kilometer Array (SKA) would be an ideal telescope to accomplish this task. The SKA would provide over a factor of 100 increase in telescope gain over the Green Bank Telescope, Parkes, and Jodrell Bank telescopes and over a factor of 30 in telescope gain over Arecibo. This improvement in gain alone would allow for deep searches, with luminosity limits less than the mean in the Faucher-Giguère \& Kaspi (2006) model, in only an hour or two for GCs less than $20 \mathrm{kpc}$ away (see Smits et al. 2009 for pulsar work with the SKA). Before the advent of the SKA, MeerKAT, the South African SKA precursor, will be able to reduce the minimum detectable flux density limit by a factor of 10 when completed (Booth et al. 2009).

This work was supported by NSF grant AST-0907967 and a WVEPSCoR Research Challenge Grant. We thank the scientific editor and anonymous referee for their help and comments during the submission process.

\section{REFERENCES}

Bailes, M., Zhu, J., \& Richter, S. 2005, in ASP Conf. Ser. 328, Binary Radio Pulsars, ed. F. A. Rasio \& I. H. Stairs (San Francisco, CA: ASP), 195

Biggs, J. D., \& Lyne, A. G. 1996, MNRAS, 282, 691

Booth, R. S., de Blok, W. J. G., Jonas, J. L., \& Fanaroff, B. 2009, arXiv:0910.2935

Briley, M. M., Bell, R. A., Hesser, J. E., \& Smith, G. H. 1994, Can. J. Phys., 72, 772 
Camilo, F., \& Rasio, F. A. 2005, in ASP Conf. Ser. 328, Binary Radio Pulsars, ed. F. A. Rasio \& I. H. Stairs (San Francisco, CA: ASP), 147

Chandler 2003, PhD thesis, Cal Tech Institute of Technology

Cordes, J. M., \& Lazio, T. J. W. 2002, arXiv:astro-ph/0207156

D’Amico, N., Bailes, M., Lyne, A. G., et al. 1993, MNRAS, 260, L7

Deneva, J. S., Cordes, J. M., \& Lazio, T. J. W. 2009, ApJ, 702, L177

Dessart, L., Burrows, A., Ott, C. D., et al. 2006, ApJ, 644, 1063

Faucher-Giguère, C.-A., \& Kaspi, V. M. 2006, ApJ, 643, 332

Ferraro, F. R., Dalessandro, E., Mucciarelli, A., et al. 2009, Nature, 462, 483

Fregeau, J. M., Richer, H. B., Rasio, F. A., \& Hurley, J. R. 2009, ApJ, 695, L20

Freire, P. C., Camilo, F., Lorimer, D. R., et al. 2001, MNRAS, 326, 901

Freire, P. C., Gupta, Y., Ransom, S. M., \& Ishwara-Chandra, C. H. 2004, ApJ, 606, L53

Freire, P. C. C. 2005, in ASP Conf. Ser. 328, Binary Radio Pulsars, ed. F. A Rasio \& I. H. Stairs (San Francisco, CA: ASP), 405

Freire, P. C. C., Ransom, S. M., Bégin, S., et al. 2008, ApJ, 675, 670

Gnedin, O. Y., Zhao, H., Pringle, J. E., et al. 2002, ApJ, 568, L23

Harris, W. E. 1996, AJ, 112, 1487

Hessels, J. W. T., Ransom, S. M., Stairs, I. H., Kaspi, V. M., \& Freire, P. C. C. 2007, ApJ, 670, 363

Hobbs, G., Lorimer, D. R., Lyne, A. G., \& Kramer, M. 2005, MNRAS, 360, 974

Hui, C. Y., Cheng, K. S., \& Taam, R. E. 2010, ApJ, 714, 1149

Ivanova, N., Heinke, C. O., Rasio, F. A., Belczynski, K., \& Fregeau, J. M. 2008, MNRAS, 386, 553

Jacoby, B. A., Cameron, P. B., Jenet, F. A., et al. 2006, ApJ, 644, L113

Johnston, S., Kramer, M., Lorimer, D. R., et al. 2006, MNRAS, 373, L6

Kitaura, F. S., Janka, H.-T., \& Hillebrandt, W. 2006, A\&A, 450, 345

Klein, B., Kramer, M., Müller, P., \& Wielebinski, R. 2004, in IAU Symp. 218, Young Neutron Stars and Their Environments, ed. F. Camilo \& B. M. Gaensler (San Francisco, CA: ASP), 133

Kramer, M., Klein, B., Lorimer, D., et al. 2000, in ASP Conf. Ser. 202, Proc. IAU Colloq. 177: Pulsar Astronomy - 2000 and Beyond, ed. M. Kramer, N. Wex, \& N. Wielebinski (San Francisco, CA: ASP), 37

Krauss, L. M., \& Chaboyer, B. 2001, arXiv:astro-ph/0111597

Kroupa, P. 2002, Science, 295, 82
Leonard, P. J. T. 1989, AJ, 98, 217

Liebert, J., Bergeron, P., \& Holberg, J. B. 2003, AJ, 125, 348

Lorimer, D. R., \& Kramer, M. 2004, Handbook of Pulsar Astronomy (Cambridge: Cambridge Univ. Press)

Lorimer, D. R., Yates, J. A., Lyne, A. G., \& Gould, D. M. 1995, MNRAS, 273, 411

Lynch, R. S., \& Ransom, S. M. 2011, ApJ, 730, L11

Lynch, R. S., Ransom, S. M., Freire, P. C. C., \& Stairs, I. H. 2011, ApJ, 734 89

Lyne, A. G., Biggs, J. D., Brinklow, A., McKenna, J., \& Ashworth, M. 1988, Nature, 332, 45

Lyne, A. G., Biggs, J. D., Harrison, P. A., \& Bailes, M. 1993, Nature, 361, 47

Lyne, A. G., Brinklow, A., Middleditch, J., Kulkarni, S. R., \& Backer, D. C. 1987, Nature, 328, 399

Lyne, A. G., Manchester, R. N., \& D’Amico, N. 1996, ApJ, 460, L41

Manchester, R. N., Hobbs, G. B., Teoh, A., \& Hobbs, M. 2005, VizieR Online Data Catalog, 7245, 0

Manchester, R. N., Lyne, A. G., Camilo, F., et al. 2001, MNRAS, 328, 17

McCrea, W. H. 1964, MNRAS, 128, 147

Mieske, S., \& Baumgardt, H. 2007, A\&A, 475, 851

Paust, N. E. Q., Reid, I. N., Piotto, G., et al. 2010, AJ, 139, 476

Ransom, S. M., Hessels, J. W. T., Stairs, I. H., et al. 2005, Science, 307, 892

Ransom, S. M., Stairs, I. H., Backer, D. C., et al. 2004, ApJ, 604, 328

Ridley, J. P., \& Lorimer, D. R. 2010, MNRAS, 404, 1081

Salpeter, E. E. 1955, ApJ, 121, 161

Scalo, J. M. 1986, Fund. Cosmic Phys., 11, 1

Smits, R., Kramer, M., Stappers, B., et al. 2009, A\&A, 493, 1161

Srinivasan, G. 1989, A\&AR, 1, 209

Tauris, T. M., \& Manchester, R. N. 1998, MNRAS, 298, 625

Taylor, J. H., \& Cordes, J. M. 1993, ApJ, 411, 674

Webbink, R. F. 1985, Dynamics of Star Clusters, Vol. 113 (Dordrecht: Reidel), 541

Wolszczan, A., Kulkarni, S. R., Middleditch, J., et al. 1989, Nature, 337, 531 